\title{
Computerassistierte, CT-freie Platzierung von Hüftpfannen
}

\author{
Paul Alfred Grützner, Ulrich Langlotz, Jürgen Korber, Dietrich Schulte Bockholt, \\ Andreas Wentzensen
}

\section{Zusammenfassung}

Ziel der Hüftnavigation ist, durch Einstellung einer exakten Position der Pfannenkomponente die Komplikationsrate in der Frühphase zu senken und die Langzeitergebnisse zu verbessern. Vorgestellt wird hier ein Verfahren, das aus einer Kombination von direkt am Patienten abgetasteten und digitalisierten Landmarken mit registrierten BV-Bildern zur Bestimmung weiterer Landmarken und zur direkten Visualisierung der Implantatposition im virtuellen Röntgenbild besteht. Das Besondere an dieser Vorge- hensweise ist, dass präoperativ kein CT-Datensatz für die Navigation benötigt wird. Mit der bildwandlergestützen Pfannennavigation können, mit gleicher Genauigkeit wie die CT-gestützte Navigation, die Inklination und Anteversion bestimmt werden. Die CT-Strahlenbelastung entfällt, die zusätzliche, zur Navigation benötigte Zeit ist kurz und die Anwendung ist sehr benutzerfreundlich. Ein wesentlicher Vorteil liegt darin, dass auf Übertragen präoperativer Daten auf die Anatomie des Patienten, das so genannte Matching, völlig verzichtet werden kann.

\section{Einleitung}

Weltweit werden jährlich etwa eine Million künstliche Hüftgelenke implantiert, in Deutschland etwa 100 000. Mit der Implantation des Gelenkes verbessert sich die Lebensqualität der Patienten in der Regel zunächst erheblich. Komplikationen durch Luxationen, Lockerungen oder Infekte führen allerdings zu einem Leidensweg, der in einem Verlust des Gelenkes enden kann. Nur die Kombination von bewährten Endoprothesenmodellen, einer exakten Implantationstechnik und der lebenslangen Betreuung der Patienten führt zu guten Langzeitergebnissen mit Überlebensraten der Prothesen von bis zu $98 \%$ nach 10 Jahren.

Die exakte Platzierung der Pfannenkomponente ist einer der wichtigsten Schritte beim korrekten Einbringen der Hüfttotalendoprothese. Die Position der Pfanne

OP-JOURNAL 2002; 17: 30-34

(c) Georg Thieme Verlag Stuttgart · New York hat Einfluss sowohl auf die kurzfristigen als auch die langfristigen Ergebnisse. Eine falsche Pfannenposition führt in der Frühphase zu einer erhöhten Luxationsrate, auf Dauer durch unphysiologische Belastung zur vorzeitigen aseptischen Auslockerung (Abb.1) [10,12].

Die Ausrichtung der Pfanne bezüglich Anteversion und Inklination ist der Schlüssel zu einer korrekten Implantation. Verschiedene Autoren haben die Komplikationsrate mit der Pfannenausrichtung korreliert und hieraus so genannte sichere Zonen der Pfannenplatzierung abgeleitet. Sie konnten zeigen, dass Hüftpfannen, die in diesem Toleranzbereich implantiert wurden, signifikant geringer zu Subluxationen und Luxationen neigen $[5,8,9,13]$. Durch eine biomechanisch ungünstige Pfannenplatzierung kann es zu verstärkten Polyethylenabrieb und somit zur frühzeitigen Lockerung kommen [6].

Ziel der Hüftnavigation ist durch Einstellung einer exakten Position der Pfannenkomponente die Komplikationsrate in der Frühphase zu senken und die Langzeitergebnisse zu verbessern.

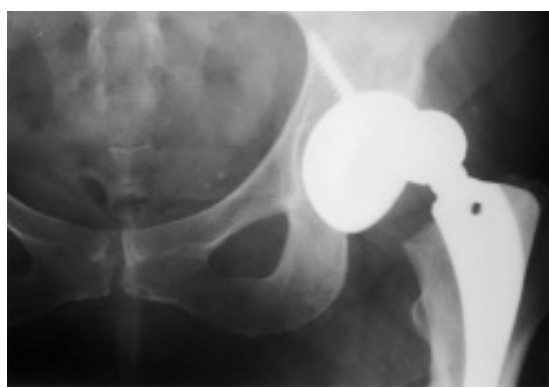

Abb.1 In Subluxation stehende Endoprothese.

Herkömmlich erfolgt die Planung beim Hüftgelenkersatz mittels Schablonen und Röntgenbild in einer 2D-Ebene. Hiermit können zwar die Inklination und die Größe der Prothese gut abgeschätzt, die korrekte Anteversion kann jedoch nicht bestimmt werden. Die genaue Position des Patienten auf dem Operationstisch ist nur schwierig abzuschätzen und bei bestimmten anatomischen Gegebenheiten wie z.B. Hüftbeugekontraktur oder Hyperlordose nahezu unmöglich. Mechanische Navigationshilfen orientieren sich hierbei mehr oder weniger am Operationstisch oder einer vom Operateur abgeschätzten Beckenlage und nicht zu einer anatomisch definierten Ebene. Extrem erschwerend ist die Relativbewegung des Beckens während der Operation. Ziel ist daher, die exakte Position der Pfannenkomponente im Becken mit einer bildgestützten Navigationshilfe zu erreichen.

In der ersten Generation von Navigationssystemen wird zur Planung und zur Visualisierung der Pfanne ein präoperatives $\mathrm{CT}$ verwandt. Obwohl die CT-basierte Navigation in vielen Fällen erfolgreich klinisch angewandt werden konnte $[1,4]$, gibt es jedoch nicht unerhebliche Nachteile und Einschränkungen. Zum einen ist ein präoperativer CT-Datensatz erforderlich, der gerade bei jüngeren $\mathrm{Pa}$ tienten eine beachtenswerte Strahlenexposition darstellt. Der Datensatz muss 
präoperativ zur Planung der Navigation aufwändig bearbeitet werden.

Hierdurch werden strukturelle und personelle Ressourcen der Klinik beansprucht. Außerdem unterliegt die CT-basierte Navigation erheblichen Limitierungen. Artefakte durch einliegende Metallimplantate oder Endoprothesen reduzieren die Bildqualität. Solche Datensätze können für die Navigation nicht verwendet werden. Sowohl die CT-basierte Planung als auch der Abgleich des CT-Datensatzes mit der Patientenanatomie intraoperativ, das so genannte Matching, unterliegen einer deutlichen Lernkurve mit Fehlermöglichkeiten.

\section{Funktionsprinzip}

Vorgestellt wird hier ein CT-freies Verfahren, das aus einer Kombination aus direkt am Patienten abgetasteten und digitalisierten Landmarken, mit registrierten BV-Bildern zu Bestimmung weiterer Landmarken besteht. Ein weiteres Merkmal besteht in der Möglichkeit der direkten Visualisierung der Implantatposition im virtuellen, navigierten Röntgenbild [7].

Ein Hybridsystem verbindet die Möglichkeit, Landmarken am Patienten direkt abzugreifen mit den Informationen aus navigierten BV Bildern.

Ausgangspunkt ist zunächst die Definition einer anatomischen Bezugsebene zu der die Pfannenposition in Relation gesetzt werden kann. Die ideale Bezugsebene ist die, von Cunningham 1922 beschriebene anatomische Beckenebene, die durch 4 anatomische Landmarken, die Spinae iliacae anteriores superiores und die Tubercula pubica, definiert wird [2,6]. Diese Ebene steht beim Gesunden im Stehen annähernd in der Frontalebene.

Durch die Definition einer anatomischen Ebene ist die Position der Pfanne reproduzierbar und unabhängig von Einflussfaktoren. Die wesentlichen Einflussfaktoren sind die Lagerung des Patienten mit Verkippung des Beckens in allen Ebenen, Hüftbeugekontraktur auf der Gegenseite und Fehlstellung in der Wirbelsäule (Abb. 2).

Die anatomische Beckenebene wird durch beide Spinae iliacae anteriores superiores und beide Tubercula pubica definiert.

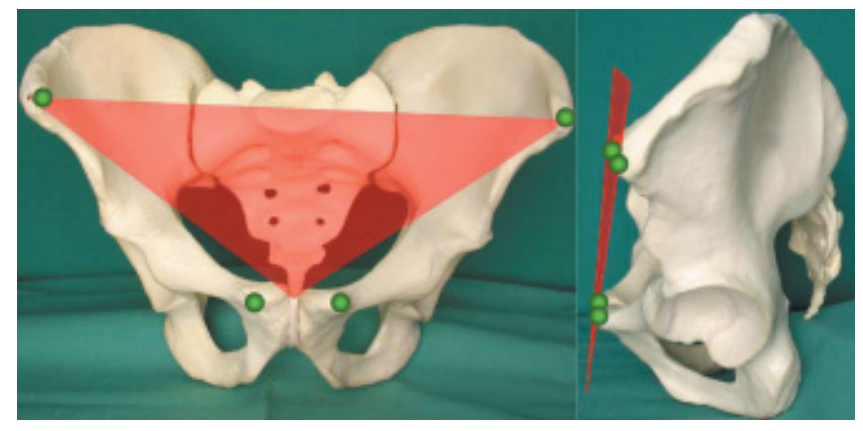

Abb. 2 Anatomische Beckenebene.

Die notwendigen Landmarken zur Definition der Patientenbeckenebene werden durch perkutanes Aufsuchen der Spinae mit einem referenzierten Pointer einerseits und durch Bestimmung des geometrischen Zentrums der Tubercula pubica mit dem Bildwandler bestimmt. Insgesamt sind hierzu 3 Bildwandlerschüsse notwendig. Das Navigationssystem errechnet aus den anatomisch definierten Punkten und den in den navigierten BV Schüssen definierten Punkten die anatomische Beckenebene und zeigt auf dem Bildschirm in „real time“ die Position, die Art und die Größe des navigierten Instruments sowie die Winkel von Inklination und Anteversion an.

Die bildwandlergestützte Pfannennavigation ist in der Lage, mit annähernd gleicher Genauigkeit wie die CT-gestützte Navigation die Inklination und Anteversion zu bestimmen. Die CT-Strahlenbelastung entfällt, die Navigationszeit ist kurz und die Anwendung ist sehr benutzerfreundlich. Ein wesentlicher Vorteil liegt darin, dass auf Übertragen präoperativer Daten auf die Anatomie des Patienten, das so genannte Matching, völlig verzichtet werden kann.

Unter registrierungsfreie Navigation versteht man Navigation auf der Basis intraoperativ erhobener Daten ohne die Notwendigkeit, ein Matching durchzuführen (einen Datensatz aktiv mit der Anatomie des Patienten abgleichen).

\section{Bestimmung der Beckenebene}

Wie oben erwähnt definiert sich die vordere Beckenebene durch die beiden Spinae iliacae anteriores und den Mittelpunkt der Verbindungslinie zwischen beiden Tubercula pubica. Mit dem vorgestellten System lassen sich anatomische Punkte prinzipiell durch zwei verschiedene Verfahren digitalisieren. Landmarken können durch direktes Abnehmen mit einem referenzierten Pointer digitali- siert werden, das heißt der Operateur definiert die Struktur nach den offensichtlich gegebenen anatomischen Verhältnissen [3]. Landmarken können aber auch mit dem navigierten Röntgenbildverstärker definiert werden. Hierzu sind BV-Einzelbilder in zwei Ebenen, die in das System geladen werden, erforderlich. In diesen registrierten BV-Bildern, die durch den kalibrierten Entzerrungsalgorithmus dem dreidimensionalen Raum geometrisch exakt zugeordnet sind, lassen sich knöcherne Landmarken definieren. Es ist prinzipiell möglich, jede Landmarke mit dem Pointer oder mit dem navigierten BV zu bestimmen [7,11].

Das Navigationssystem verknüpft die Information der Fräser- und Pfannenposition als Anzeige in Winkelgraden der Inklination Antetorsion und in Relation zur anatomischen Beckenebene. Durch die Registrierung sowohl des Patienten über die dynamische Referenzbasis und der Instrumente ist dies völlig unabhängig von der Lage des Patienten. Zusätzlich werden die Bildinformationen aus den BV-Bildern in das System transferiert und im Sinne der virtuellen Fluoroskopie mit den Informationen der Instrumente verknüpft. Der Operateur hat also nicht nur die reinen Winkelinformationen, sondern zusätzlich Informationen zur Frästiefe, Pfannenüberdachung und zum Hüftzentrum.

Ein weiterer entscheidender Vorteil der intraoperativen Datengewinnung ist die Möglichkeit, jederzeit die Genauigkeit des Systems zu überprüfen, bei Änderungen der Anatomie durch Pfannendachplastiken oder bei Revisionseingriffen neue BV-Bilder in das System zu laden und mit den jeweils aktuellsten Bildinformationen die Navigation durchzuführen.

\section{Operationsablauf}

Um eine navigierte Pfannenimplantation durchzuführen, können die Operations- 
abläufe nahezu unverändert beibehalten werden. In unserer Klinik erfolgt der Zugang standardmäßig transgluteal, modifiziert nach Bauer. Nach Resektion und Extraktion des Hüftkopfes und Vorbereitung der Pfanne zum Fräsen wird am Becken des Patienten eine dynamische Referenzbasis (DRB) befestigt. Dies erfolgt entweder supraazetabulär durch den Operationszugang oder über eine gesonderte Stichinzision an der Spina iliaca. Es ist unbedingt auf eine stabile Verankerung der DRB zu achten, da jede Dislokation der DRB bei der eigentlichen Navigation zu einer fehlerhaften Anzeige führt (Abb. 3).

Dynamische Referenzbasis: Ausgleich der Relativbewegungen zwischen Patient und Instrumenten. Stabile Fixierung erforderlich.

Im nächsten Schritt wird nun die anatomische Beckenebene des Patienten bestimmt. Dies ist erforderlich, um eine konstante, lageunabhängige Referenzebene für die Position der Pfanne zu definieren. Die anatomische Beckenebene definiert die Frontalebene des Beckens im Stehen und dient gleichzeitig als Referenzebene für postoperative Kontrollen. Die Spinae lassen sich intraoperativ in der Regel gut tasten und können daher einfach perkutan mit dem Pointer digitalisiert werden. Demgegenüber ist der direkte Zugang zu den tubercula pubica nicht möglich. Die Tuberkula werden daher in der Becken Inlet und Outlet-Projektion, zentriert auf die Symphyse in BV-

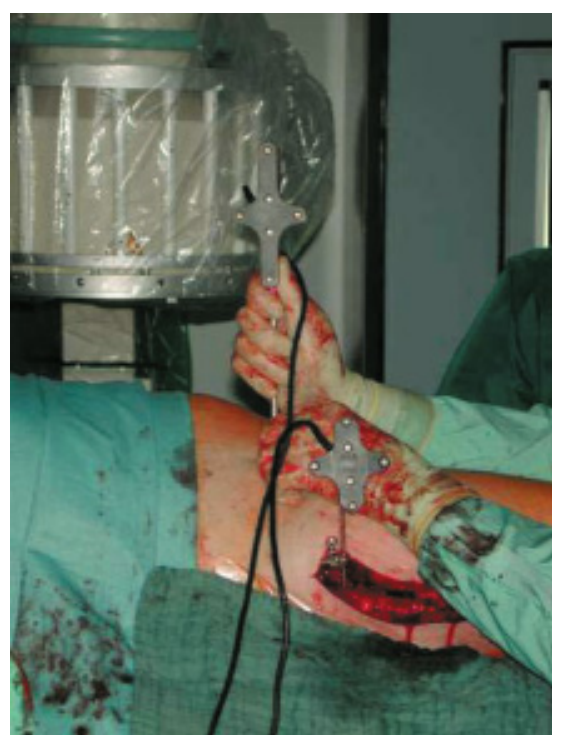

Abb. 3 Dynamische Referenzbasis supraazetabulär fixiert, navigierter Pointer auf der Spina ant. sup.

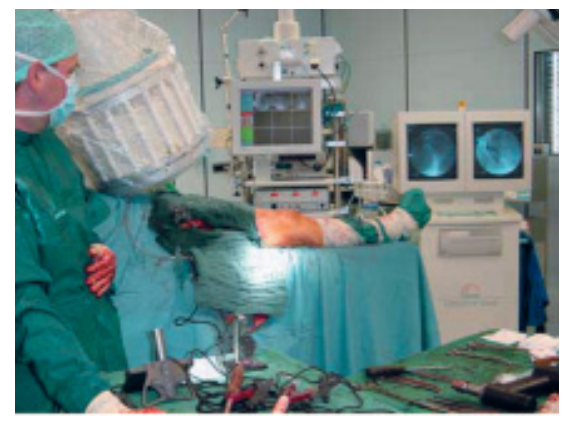

Abb. 4 a Inlet Aufnahme der Symphyse.

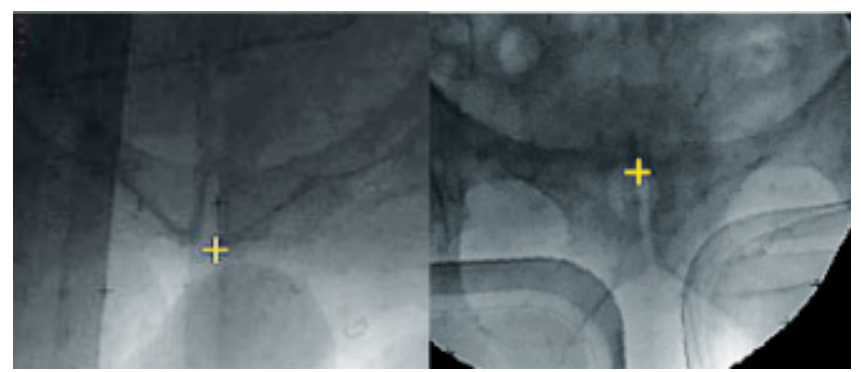

Abb. 4b Inlet und Outlet Projektion.

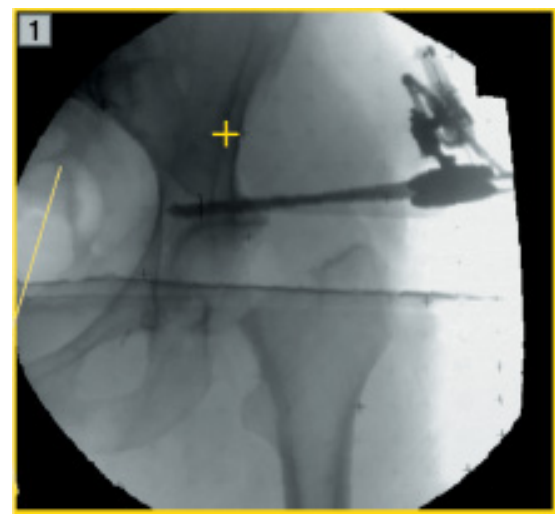

Abb. 5 A.-p.-Aufnahme des Azetabulums.

Einzelbildern aufgenommen und die Bilder in das System geladen. Die BV Bilder sollten mindestens in $60^{\circ}$ unterschiedlichem Projektionswinkel aufgenommen sein. In diesen, entsprechend dem Kalibrierungsmodus entzerrten Bildern erfolgt die geometrisch exakte Definition des Mittelpunktes der Verbindung beider Tubercula. Ein weiteres Bild wird vom Aketabulum in A.-p.-Projektion aufgenommen und in das System geladen (Abb.4u.5).

Nach Befestigung der DRB, Aufnahme der Röntgenbilder und Definition der Beckenebene können das Auffräsen der Pfanne und das Einbringen des Implantates erfolgen. Der Operateur erhält die Informationen aus dem Navigationssystem zusätzlich zum gewohnten Operationsablauf. Es ist jederzeit möglich, die Plausibilität zu überprüfen und die Genauigkeit des Systems über zuvor festgelegte
Konfidenzpunkte zu verfolgen. Sowohl beim Fräsvorgang als auch beim definitiven Platzieren der Pfanne werden die aktuellen Inklinations- und Antetorsionswinkel in Echtzeit angezeigt. Zusätzlich wird die Position der Instrumente in den virtuellen BV-Bildern angezeigt. Damit erhält der Operateur zusätzliche Informationen zur Tiefe, der Überdachung am Pfannenerker und zur Zentrierung der Pfanne. Nach der endgültigen Platzierung der Pfanne werden die Winkel und die Pfannenposition gespeichert und die Operation wird konventionell weitergeführt. Der zum konventionellen Vorgehen zusätzliche Zeitaufwand ergibt sich daher aus Anbringen und Entfernen der DRB, dem Aufnehmen der BV-Bilder und der Definition der Beckenebene und beträgt in unserer Untersuchung weniger als 10 Minuten ohne wesentliche Lernkurve.

Der Operateur kann alle Vorgänge im Navigationssystem über ein so genanntes virtuelles Keyboard mit der vollen Funktionalität einer Computermaus, der „virtuellen Maus“, vom OP-Tisch aus steril bedienen. Die Kamera erkennt hier die Position der Bedienungsplatte in Relation zur Spitze eines Instrumentes (Abb.6-8).

\section{Ergebnisse}

Zwischen Februar 2001 und Februar 2002 wurden in unserer Klinik bei 100 Patienten Hüftgelenkpfannen unter Verwendung der CT-freien Navigation implantiert. Darunter waren 20 Patienten mit Pfannenrevisionen aufgrund aseptischer Lockerungen. Die angestrebten 

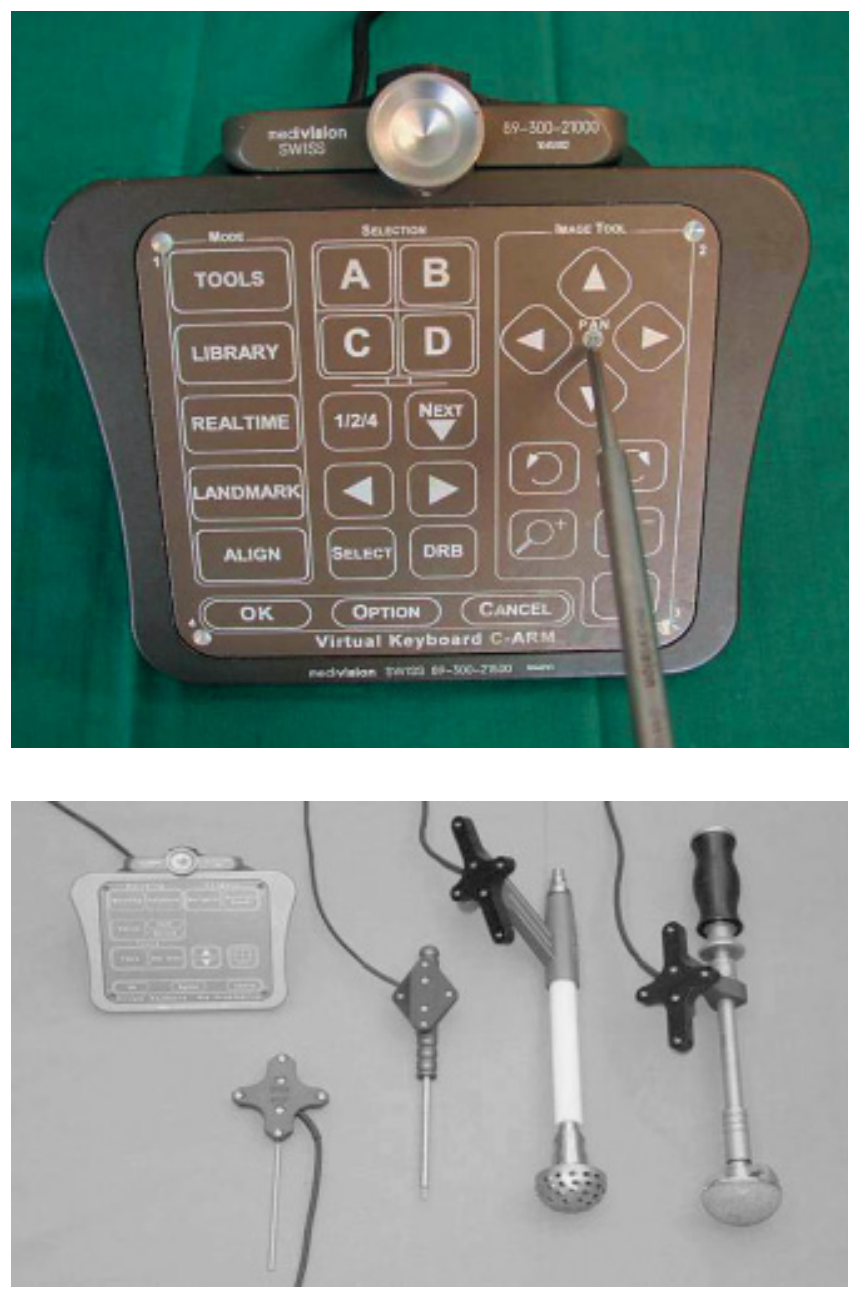

Abb. 7 Zur Navigation mit LED-Markern versehenes Instrumentarium (virtuelles Keyboard, dynamische Referenzbasis, Pointer, Fräder, Einschläger mit Implantat).

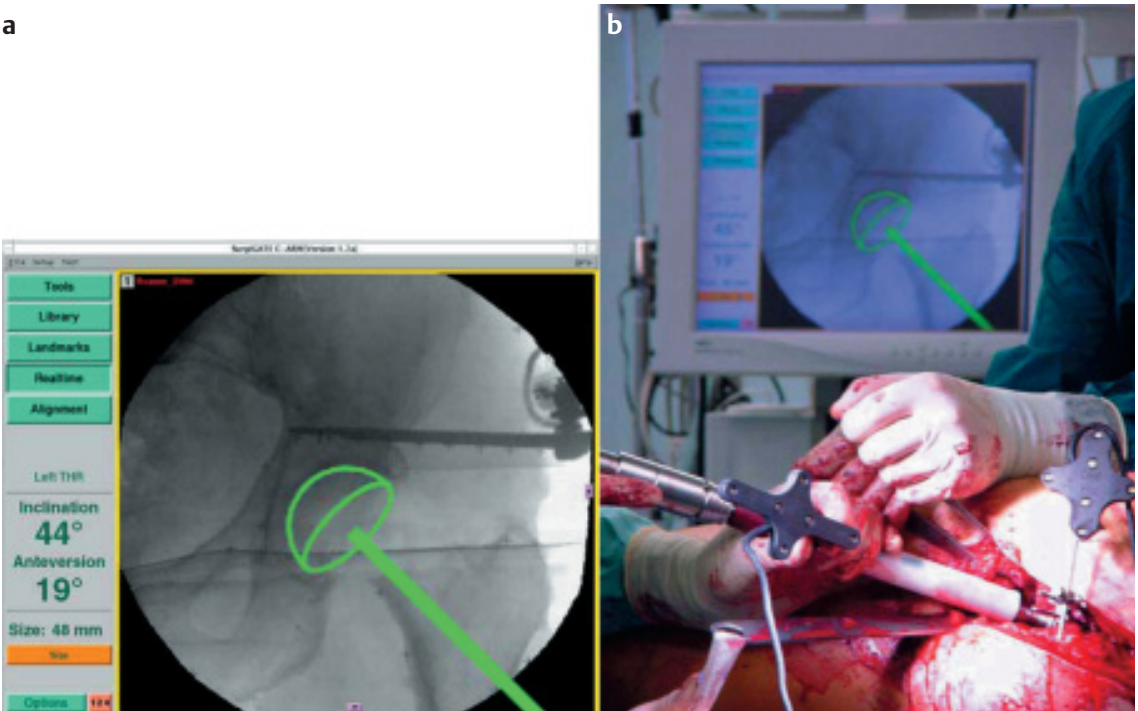

Abb.8au.b Pfannenplatzierung mit Anzeige der Winkelgrade und Projektion der Instrumentenposition in das virtuelle Röntgenbild.
Winkel waren $40^{\circ}-45^{\circ}$ Inklination und $15^{\circ}-25^{\circ}$ Antetorsion, abhängig von der ventralen Pfannenüberdachung.

Die Pfannen wurden in einem mittleren Inklinationswinkel von $43^{\circ}\left(39^{\circ}-48^{\circ}\right)$ und einem mittleren Antetorsionswinkel von $19^{\circ}\left(10^{\circ}-28^{\circ}\right)$ implantiert. Die mittlere OP-Schnitt- und -Nahtzeit betrug $85^{\circ}$ einschließlich der Revisionseingriffe. Die mittlere Zeit zum Einbringen der Pfanne, gemessen ab dem Anbringen der dynamischen Referenzbasis bis zum Entfernen der DRB, betrug $20^{\circ}$.

Die Zeiten zeigen, dass durch die Navigation die gesamte OP-Dauer nur unwesentlich verlängert wird und die Navigation ein sehr standardisiertes Einbringen des Implantates ohne „Ausreißer“ möglich ist. In dieser Gruppe haben wir keine Infekte, keine Luxationen oder sonstige Frühkomplikationen beobachtet.

\section{Schlussfolgerung}

Durch die hybride Navigation ist eine exakte Pfannenpositionierung beim Hüftgelenkersatz mit minimaler Änderung des normalen Operationsablaufs und ohne ein präoperatives $\mathrm{CT}$ möglich. Die Navigation ist jedoch nur eine Hilfe für den Chirurgen und ersetzt nicht mangelnde Kenntnisse und Erfahrung des Operateurs. Die Navigation kann dem Chirurgen hochpräzise die Position des Implantates in Relation zu den Informationen aus Röntgenbildern und anatomischen Punkten zeigen. Es gibt dem Chirurgen aber nicht die Vorgaben, welches die richtige Position ist. Da aber jetzt ein präzises, reproduzierbares Messinstrument zur Verfügung steht, wird es in Zukunft besser möglich sein, diese Daten mit klinischen Ergebnissen zu korrelieren. Man wird dann der Frage, welche Position des Implantates ideal für den jeweiligen Patienten ist, näher kommen.

Wünschenswert und erforderlich ist es natürlich auch den Schaft, basierend auf dem gleichen System, mit in die Navigation einzubeziehen. Nur hierdurch wird es möglich sein, durch Analyse des Impingements Bestimmung der Beinlängenänderung, Messung der Schaftantetorsion und weitere Parameter die Hüftendoprothetik weiter zu optimieren. Die Schaftnavigation steht kurz vor der klinischen Einführung. 


\section{Literatur}

${ }^{1}$ Bernsmann K, Langlotz U, Ansari B, Wiese M. [Computer-assisted navigated acetabulum placement in hip prosthesis implantation - application study in routine clinical care]. Z Orthop Ihre Grenzgeb 2000; 138: 515-521

${ }^{2}$ Cunningham P. In: Cunningham, ed Cunningham's Textbook of Anatomy. London RA: Henry Frowde and Hodder \& Stroughton, 1922: 255-260

${ }^{3}$ Dessenne V, Lavallee S, Julliard R, Orti R, Martelli S, Cinquin P. Computerassisted knee anterior cruciate ligament reconstruction: first clinical tests. J Image Guid Surg 1995; 1: 59-64

${ }^{4}$ DiGioia AM, Jaramaz B, Blackwell M et al. The Otto Aufranc Award. Image guided navigation system to measure intraoperatively acetabular implant alignment. Clin Orthop 1998; 8-22

${ }^{5}$ Harris WH. Advances in surgical technique for total hip replacement: without and with osteotomy of the greater trochanter. Clin Orthop 1980; 188-204

${ }^{6}$ Hirakawa K, Mitsugi N, Koshino T, Saito T, Hirasawa Y, Kubo T. Effect of acetabular cup position and orientation in cemented total hip arthroplasty. Clin Orthop 2001; 388: $135-$ 142
${ }^{7}$ Hofstetter R, Slomczykowski M, Sati M, Nolte LP. Fluoroscopy as an imaging means for computer-assisted surgical navigation. Comput Aided Surg 1999; 4: 65-76

${ }^{8}$ Kummer FJ, Shah S, Iyer S, DiCesare PE. The effect of acetabular cup orientations on limiting hip rotation. J Arthroplasty 1999; 509-513

${ }^{9}$ Lewinnek GE, Lewis JL, Tarr R, Compere CL, Zimmermann JR. Dislocations after total hip-replacement arthroplasties. J Bone Joint Surg Am 1978; 60: 217-220

${ }^{10}$ McCollum DE, Gray WJ. Dislocation after total hip arthroplasty. Causes and prevention. Clin Orthop 1990; 159-170

${ }^{11}$ Nolte LP, Slomczykowski MA, Berlemann U et al. A new approach to computer-aided spine surgery: fluoroscopy-based surgical navigation. Eur Spine J 2000; 9 Suppl 1: S78-S88

${ }^{12}$ Sarmiento A, Ebramzadeh E, Gogan WJ, McKellop HA. Cup containment and orientation in cemented total hip arthroplasties. J Bone Joint Surg Br 1990; 72: 996-1002

${ }^{13}$ Seki M, Yuasa N, Ohkuni K. Analysis of optimal range of socket orientations in total hip arthroplasty with use of computer-aided design simulation. J Orthop Res 1998; 16: 513 517
Dr. med. Paul Alfred Grützner

Oberarzt

Dr. med. Bernd Vock

Oberarzt

Cand. med. Jürgen Korber

AG Rechnergestütztes Operieren

Dr. med Dietrich Schulte Bockholt

Assistenzarzt

Prof. Dr. med. Andreas Wentzensen

Ärztlicher Direktor

BG-Unfallklinik Ludwigshafen

Unfallchirurgische Klinik der Universität Heidelberg

Ludwig-Guttman-Str. 13

D-67071 Ludwigshafen

\section{Dr. Ing. Ulrich Langlotz}

ME Müller Institut für Biomechanik Universität Bern

Murtenstr. 32

CH-3010 Bern 